\title{
IDENTIFICATION OF UNCERTAINTY AND QUANTIFICATION OF VARIABILITY IN WOVEN COMPOSITE MATERIALS BASED ON CARBON FIBRE WEAVES
}

\author{
Andy Vanaerschot ${ }^{1}$, Stepan V. Lomov ${ }^{2}$, David Moens ${ }^{1}$ and Dirk Vandepitte ${ }^{2}$ \\ ${ }^{1}$ KU Leuven \\ Dept. of Mechanical Engineering, Kasteelpark Arenberg 41 - box 2449 \\ e-mail: \{andy.vanaerschot, david.moens, dirk.vandepitte\} @ kuleuven.be \\ ${ }^{2}$ Dept. of Materials Engineering, Kasteelpark Arenberg 44 - box 2450 \\ e-mail: stepan.lomov@mtm.kuleuven.be
}

Keywords: Instructions, ECCOMAS Thematic Conference, Uncertainty Quantification, Multiscale Analysis, Proceedings.

\begin{abstract}
Composite materials are created as a quite complex architecture which includes a fibre reinforcement structure and matrix material. Many material parameters play a role when composite structures are modelled, e.g. in finite element models. In addition to the properties of the raw fibre and matrix materials which are used, also geometrical parameters have a significant effect on structural characteristics.

With the multitude of model parameters which have to be taken into account, the effect of uncertainty becomes even more complicated. For many parameters the range or the distribution is not well known. The objective of this paper is to develop a consistent approach to identify and to quantify the effect of uncertainty and variability as concisely as possible, in order to allow for structural analysis which takes into account variability in a realistic way.

In addition to the identification of probability distribution functions which are established, parameter correlations between different positions along a tow and between two tows are also determined.
\end{abstract}




\section{INTRODUCTION}

Composite materials are attractive for weight sensitive applications in highly loaded structural components. There are many sources of uncertainty, because of a multitude of design and process parameters which may be uncertain or variable. Typical applications like aircraft structures are subject to very strict performance requirements, implying that uncertainty needs to be taken into account in the analysis and qualification processes. The first part of the presentation gives an overview of typical sources of uncertainty which play a role in the design and analysis of composite materials. In addition to the properties of the raw materials (fibre and matrix) as such, the architecture and the geometry of the fibre reinforcement also play an important role: yarn placement and spacing, waviness, cross-sectional area, etc.

The second part of the paper focusses on the quantification of variability in composite material stiffness properties. A correct representation of the materials geometry and properties can only be achieved by (i) collecting enough experimental data on the spatially correlated random fluctuations of uncertain tow path parameters and (ii) deriving probabilistic information for the macroscopic properties from the lower scale mechanical characteristics. This work presents the general approach and successive steps to build random virtual specimen, which can subsequently be used to evaluate the spatial variation in the mechanical properties such as stiffness. The methodology is applied on a typical woven textile composite.

Experimental data are collected of the random geometrical structure of the woven textile composite. Tow path properties are analysed on the short- and long-range, i.e. spanning several unit cells. The spatial variation of each tow parameter is decomposed in non-stochastic, periodic systematic trends and nonperiodic stochastic fluctuations. The systematic trend represents the average behaviour of the tow parameter, while the stochastic characteristics are given in terms of the standard deviation and correlation length. The next step is the generation of virtual random structures possessing the same statistical properties as the experimental sample. The random tow path is obtained starting from a Monte Carlo Markov chain algorithm, originally proposed for ceramic composites, where the transition matrix of each tow component is calibrated with its experimental standard deviation and correlation length. The approach provides fluctuations of each single tow parameter using distinct Markov chains to ensure a different degree of variations on the lower and the macro-scale, as observed from experiments. A virtual specimen is obtained by combining these generated deviations with the experimental systematic trends.

This paper presents the general approach and successive steps to build random virtual specimens, which are subsequently used to evaluate the spatial variation in the mechanical properties. This work focusses on the stiffness tensor of the material with orthotropic characteristics. The proposed methodology is applied on a typical woven textile composite.

\section{STATE-OF-THE-ART IN UNCERTAINTY MODELLING IN COMPOSITES}

\subsection{Literature review}

All material properties exhibit scatter. Two types of scatter occur, in time and in space. Scatter in time is a fluctuation of mechanical properties that is due to some physical or chemical mechanism or to changing environmental conditions to which the material sample is subjected. Most of these mechanisms are well understoo. Scatter in space is the effect that material properties are not constant over the area or the volume of one and the same component in one specific material. In composites scatter is caused by differences in (1) fibre orientations and in fibre positions and fibre paths, and in (2) material processing effects, an in a later stage also by (3) the onset of fatigue damage. Examples of processing effects are deviations of organised textile 
reinforcement that are due to processing (dimensions and position of yarns, taking into account local compression of the yarns and the fibrous plies). The scale on which these differences occur is the size of a unit cell.

Probabilistic methods are used to describe scatter in properties. Probability distribution functions (pdf) can be established for all uncertain parameters, taking into account the correlation between different parameters. The result of the analysis can be interpreted in a statistical sense, and the probability of every output quantity depends on the input probabilities and their correlations. It is important that all these inputs must be validated in order for the result to allow for a statistical interpretation. Unfortunately this fact is often neglected by many scientists, and assumptions are made on the input pdfs [1]. The definition of an input pdf is then subjective, and so is the result. Freudenthal [2] states that "ignorance of the cause of variation does not make such variation random.". The availability of objective and validated data is thus required.

Scatter in material properties directly determines quality of the material, the product or the effect of a processing step. The most common application of stochastic analysis is reliability analysis of load carrying structures. This type of analysis predicts the probability of failure of a structural component, based on the pdfs of all product parameters, including material properties, geometrical characteristics and loading. It is usually done using Stochastic Finite Element analysis.

Composite materials are usually modelled in a multi-scale approach. This research considers the meso- and the macro-scales.

\subsection{State-of-the-art in stochastic modelling for material behaviour and material process- ing}

The research community has already spent much work on stochastic characteristics of composite materials. The texture is described by Langelaan et al. [3]. Zeman [4] and Sejnoha [5] have developed a mathematical framework for the determination of the unit cells of plain weave fabric composites with reinforcement imperfections, and they treat imperfections in a combined manner. Desplentere et al. [6] have developed an integrated approach for the simulation of the RTM process with uncertain permeability over the volume of the textile reinforcement. Endruweit et al. [7] and Markicevic et al. [8] have used a similar technique to predict the quality variations in components that are processed by RTM. They are able to predict the probability of dry spots in the component. This analysis is required in the development of a robust process with stable quality.

Almost all the published work deals with randomness of local properties, without taking into account the correlation of properties between different positions in a component. Knowledge of this correlation is however essential to correctly model spatial distribution of properties, as that affects the global behaviour of the component. The numerical formalisms that are needed for the correct description of the spatial scatter with correlation are already available for a long time. The concept of random fields $[9,10]$ can be seen as a two- or three-dimensional extension of a traditional scalar or vector stochastic process. In the application of material properties, a parameter value at one position in the material is stochastic, but its variation with the two (thin plate) or three (thick plate or volume) dimensions of the components has to be considered as well. The Karhunen-Loève decomposition expresses the spatial distribution of the random variable as a summation of well-chosen basis functions that are each multiplied by a random weighting factor. The basis functions are chosen such that for Gaussian stochastic processes the random coefficients are uncorrelated and thus independent random variables. These functions further have the appropriate mathematical properties to improve numerical performance. The 
basis functions are calculated from an eigenvalue problem on the covariance matrix. The covariance matrix expresses the correlation between values of the parameter that is considered at two different positions. It thus plays a central role in the description of spatial scatter. In conjunction with stochastic finite elements, an exponential function is often used for the covariance kernel. An essential parameter is the correlation length. A realistic value of correlation length is required for the random field to correctly describe the stochastic behaviour of the modelled parameter.

Significant contributions have been made in the development of material models with uncertain parameters. Chung and de Borst [11] developed a stochastic simulation of fibre metal laminates (Glare). In their work, they make assumptions on the randomness of the modulus of the composite layer.

There is now a severe lack on practical data on real material systems and on concepts to generate reliable and useful data in a format that can be fed into the powerful numerical models that are available. All authors content themselves with making assumptions, e.g. on the covariance function of the Young's modulus and the correlation length. They have done sensitivity analyses and they prove that the correlation length has a very large effect on the final result, yet validated data are not available. Charmpis et al. [12] propose two approaches to achieve significant advances in realistic material modelling : (1) establishing experimental data on the spatially correlated random fluctuations of uncertain material properties, and (2) deriving probabilistic information for macroscopic properties from the lower scale mechanical characteristics of materials. Although the need for experimental data was identified already in the 80ies, it appears that no evident step in this direction has been made until a few years ago. The second approach is essentially a multi-scale approach.

\subsection{Effect of parameter scatter on material stiffness properties}

Composite materials are attractive because they offer wide opportunities for tailored solutions in addition to excellent mechanical performance. The designer has many degrees of freedom, including the selection of raw materials for both the matrix and the fibre reinforcement, the architecture of the fibre reinforcement (unidirectional fibres, woven fabrics, knitted fabrics, braided fabrics, non-crimp fabrics, ..., each with its own variants), the fibre volume fraction, the number of layers and the orientation of layers. For the analyst, this large set of design degrees of freedom translates into a wide range of model parameters, and inevitably also a wide range of uncertain or imprecise material data.

Figure 1 illustrates some of these effects. The left hand side of the figure shows the variation of the elastic orthotropic stiffness constants for different orientations of a uniaxially reinforced glass fibre composite lamina with respect to the applied uniaxial tensile load. $E_{11}$ is the modulus in the longitudinal direction along the fibre orientation, and $E_{x x}$ is the modulus in the loading direction that has an angle $\theta$ with respect to the fibre direction. The graph shows a significant decrease of stiffness with increasing misalignment of the fibre. The right hand side of the figure is valid for a cross-ply $\left(0^{\circ}-90^{\circ}\right)$ carbon-epoxy system. The graph shows the variation of the Young's modulus for different alignments of the the fibre orientations with respect to the loading direction. The graphs show that the equivalent material stiffness depends strongly on the fibre placement. An imprecise placement of the fibre inevitably leads to a change of stiffness with respect to the nominal values. The left hand side of the graph also shows that the orthotropic elastic constants are inter-related.

Another geometrical parameter that determines the homogenised stiffness characteristics of a textile composite material is the so-called crimp factor. It is a measure of the waviness of the 

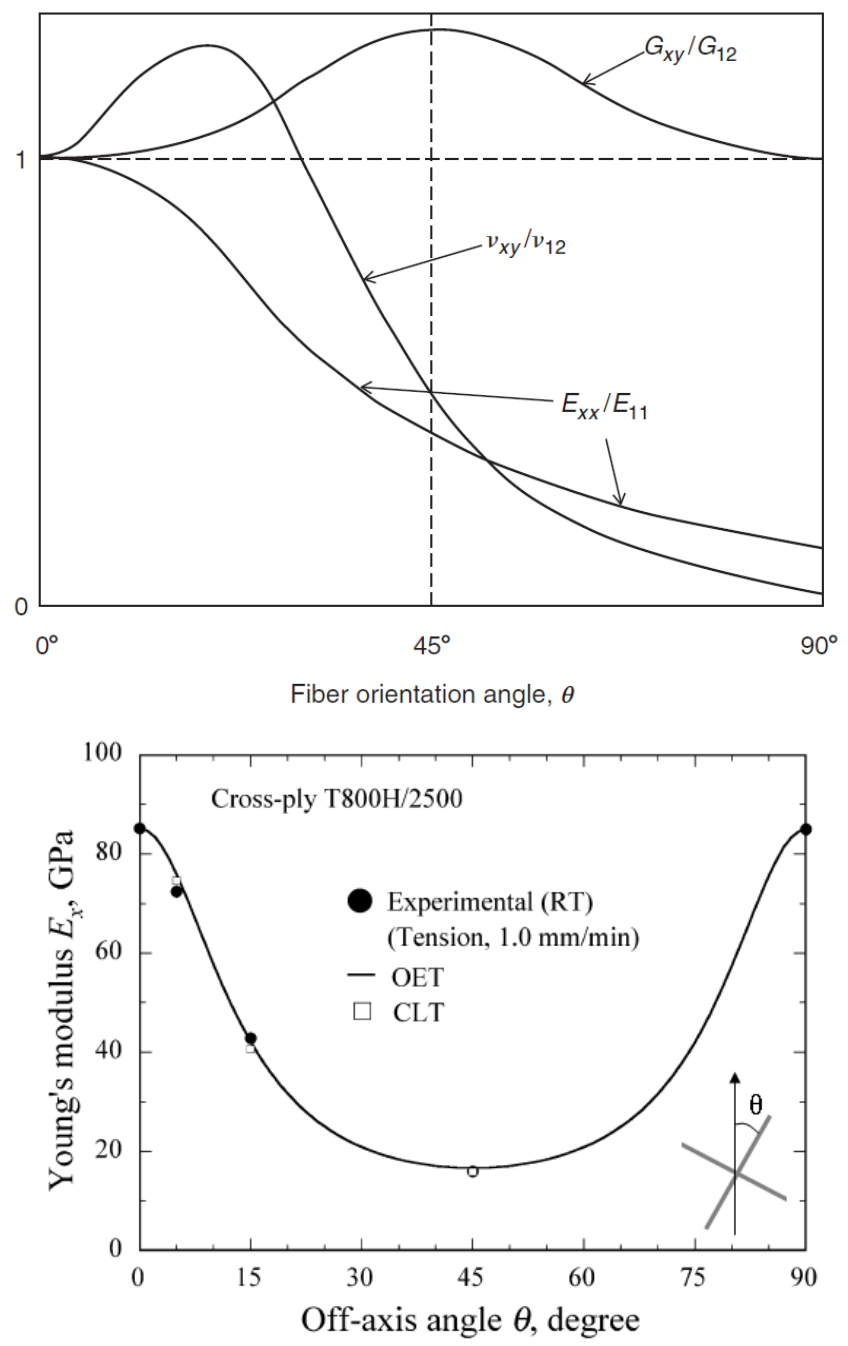

Figure 1: Dependency of in-plane material parameters on the orientation $\theta$ of the major fibre axis to the loading direction; top: variation of the elastic constants of a continuous E-glass fibre lamina [13]; bottom: variation of the tensile Young's modulus for a cross-ply carbon-epoxy composite [14]

yarn through the thickness of the panel. A general tendency is that the equivalent modulus of a textile composite increases with decreasing crimp.

\subsection{Multi-scale models for spatial variation of material properties}

Research has brought forward significant advances in models that describe different aspects of material non-homogeneity. Extensive research efforts are currently ongoing to develop a multi-scale modelling procedure at successive scales. Depending on the type of material, the micro-scale describes properties with a reference length in the order of $10^{-6}-10^{-4} \mathrm{~m}$, the meso-scale describes properties with a reference length in the order of $10^{-4}-10^{-2} \mathrm{~m}$, and entire component structural behaviour is described on the macro-scale, with reference lengths in the order of $10^{-2}-10^{0} \mathrm{~m}$ and above. The step from a lower level to a higher level is made using homogenisation procedures, that assign overall properties at a higher scale based on lower scale data. So far, these models are mainly deterministic. When these models will be well established, they present an excellent opportunity to introduce variability at the appropriate level, and to predict the propagation of their effect to a higher level, and ultimately to the entire component. 
Charmpis and Schuëller [12] have already made proposals to materials researchers to develop these models. Experiments will however always be required to validate these models.

Multi-scale models also have the advantage that spatial variation of homogenised properties can be described based on lower scale characteristics. This presents opportunities for realistic quantification of random fields, for which experimental data are currently missing.

\section{QUANTIFICATION OF VARIABILITY ON COMPOSITE GEOMETRY IN CAR- BON FIBRE WEAVES}

The objective of this section is to develop a consistent modelling strategy which can be used to generate virtual samples of a composite reinforcement architecture. The statistics of the set of samples which are generated should match the statistics of a typical real composite panel. Figure 2 summarises the entire procedure.

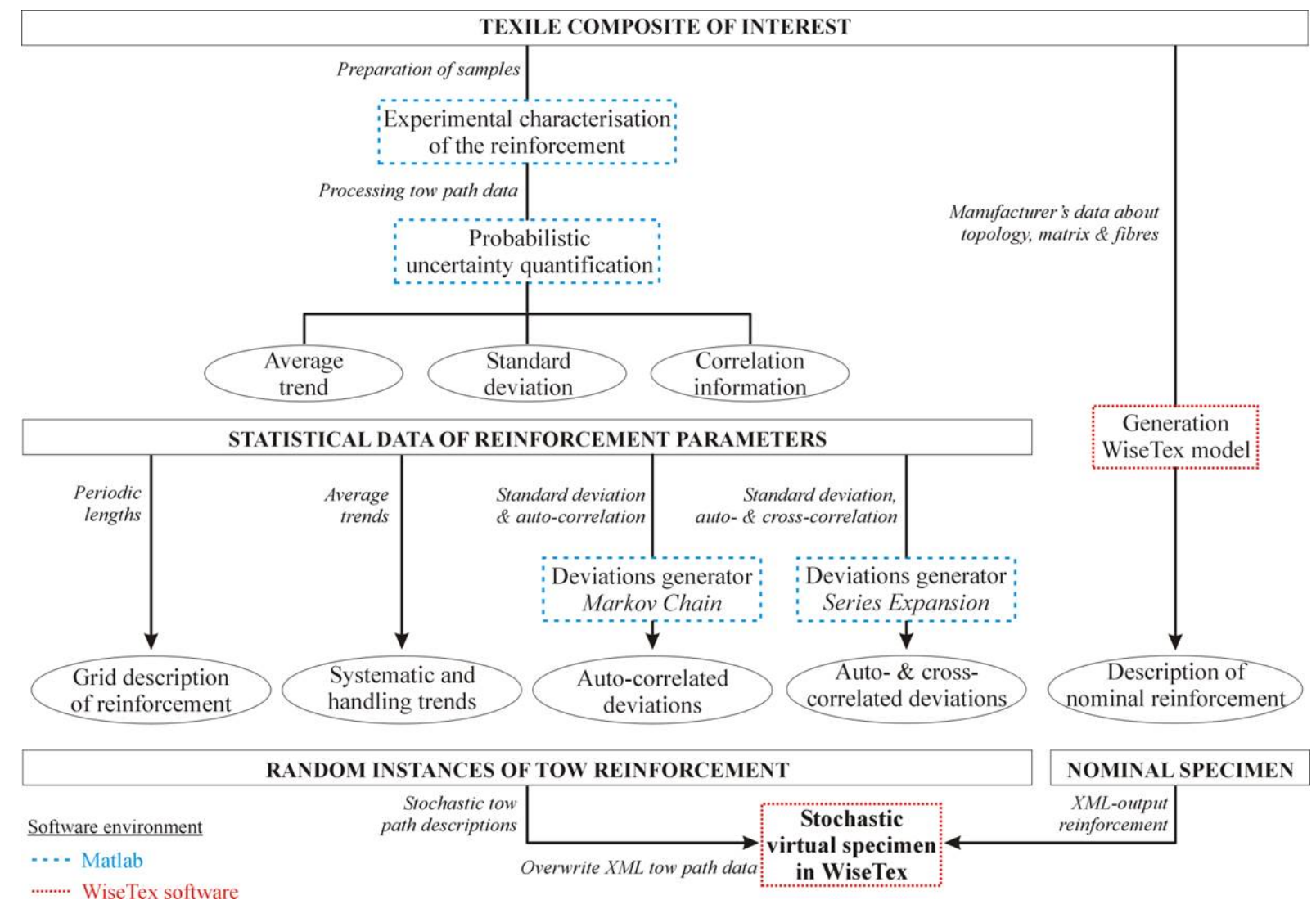

Figure 2: The general methodology for modelling data uncertainty in composite reinforcement geometry consists of three steps: 1- acquisition of geometry data; 2- processing of statistical data and correlation within one tow and between adjacent tows; 3 - generation of virtual tow realisations which have the same statistics as the experimental data set

\subsection{Modelling strategy}

Realistic woven specimens are acquired that are replicas of experimental samples. Randomness is introduced in the numerical models at the meso- and macro-level; scatter in the matrix and fibre properties are not considered. Variability of each tow path is defined for the centroid co-ordinates $(x, y, z)$, tow aspect ratio $A R$, tow area $A$ and orientation $\theta$ in cross-section which fully describe a woven reinforcement. Figure 2 presents an overview of the multi-scale 
framework, where three main steps can be distinguished to obtain such random representations:

1. The first step is the collection of experimental data and the subsequent statistical analysis, according to the procedure proposed by Bale et al. [15]. Two length scales are considered, one on the level of the representative volume element using $\mu \mathrm{CT}$ and the other on the scale of multiple unit cells. The former set gives precise positional and cross-sectional data on the meso-level, identifying full details of 3D geometry. The latter data set is required to describe long-scale deviations from the nominal reinforcement pattern.

(a) Characterisation of the short-range scatter (meso-scale) with samples close to the unit cell size.

(b) Characterisation of the long-range variation (macro-scale) with samples spanning several unit cells.

(c) Statistical analysis of the tow path parameters in terms of average trends, standard deviation and correlation lengths. For each parameter, the average trend and the local deviation are separated:

$$
\epsilon_{i}^{j, t, p}=\left\langle\epsilon_{i}^{j, t, p}\right\rangle+\delta \epsilon_{i}^{j, t, p}
$$

where $i$ refers to the location, $j$ to the tow number, $t$ to the tow genus (warp or weft) and $p$ to the ply or sample.

Vanaerschot et al. [16] give full details on the analysis procedure and statistical data processing.

2. The second step is the stochastic multi-scale modelling of the reinforcement architecture that is experimentally identified in step 1.

(a) Definition of systematic and handling trends from the experimental data.

(b) Generation of zero-mean deviations correlated along the tow path using the Monte Carlo Markov chain method.

(c) Generation of zero-mean deviations correlated along and between neighbouring tow paths using the cross-correlated series expansion technique.

For the generation of geometry data, two options are available. When cross-correlation between adjacent tows are not taken into account, the procedure by Blacklock et al. [17] is used. The representation is done using a Markov process. Each tow parameter is generated in an independent way, and for the calibration step, standard deviation and the nearest neighbour correlation information is used. When cross-correlations are taken into account, a cross-correlated series expansion is used, based on Karhunen-Loève decomposition, as proposed by Vorechovský et al. [18, 19]. Vanaerschot et al. [20] give full details on the generation of an approximated random field.

3. Construction of virtual specimens in the WiseTex software

(a) Simulation of the nominal model with matrix and fibre properties from the manufacturer.

(b) Redefinition of the reinforcement information with the produced tow paths. 


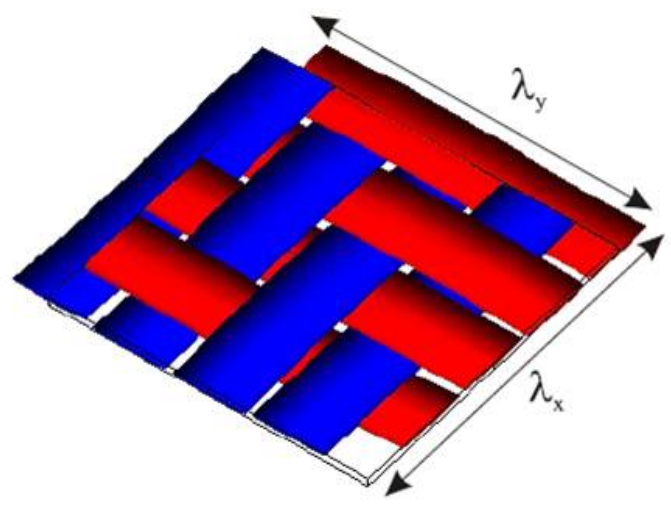

Figure 3: WiseTex model of a twill $2 / 2$ unit cell

(c) Recalculations of the path orientation vectors and length, in addition to the updated general unit cell properties.

The WiseTex [22] software suite is developed at the Materials Engineering Department of KU Leuven. It is a pre-processor for the generation of virtual 3D models of a wide range of textile composite architectures. The pre-processor prepares for the generation of finite element models for the mechanical analysis of composite structures.

This step of the analysis generates virtual specimens which have the same statistical characteristics as the samples on which the experimental data acquisition was done. For each virtual sample, a WiseTex model is generated, with the original nominal tow path being overwritten by updated paths. Discrete random tow path realisations are interpolated and accompanied with information on the orientation vectors of each tow cross-section, the path length for each segment between two discrete locations and with new, off-nominal unit cell dimensions and properties.

Vanaerschot et al. [21] present details of the procedure for generating virtual samples.

The entire methodology allows for the generation of a set of statistically relevant samples. In a subsequent analysis step, these models are used for the investigation of the homogenised properties of a composite panel.

When other topologies than woven are considered, additional parameters should be quantified to allow a full description, e.g. the braid angle for braids and the distortion of the $z$-yarn in case of non-crimp fabrics.

\subsection{Application to a carbon fibre twill weave}

This section presents an application to the methodology which is described above.

\subsubsection{Sample data}

The entire procedure is applied on a polymer textile composite with a twill $2 / 2$ woven topology (Hexcel@G0986 Injectex), with 6K carbon fibre AS4C tows. The matrix is an expoxy resin Epikote $\AA 828$ LVEL with Dytek $囚 D C H-99$ hardener. The samples are prepared in a Resin Transfer Moulding (RTM) process and the unit cell size is $11.4 \mathrm{~mm} \times 11.4 \mathrm{~mm}$. Figure 3 shows a virtual WiseTex model of one unit cell of the sample on which data have been collected. Some of the samples on which data have been acquired have 7 layers, others have only a single layer. 


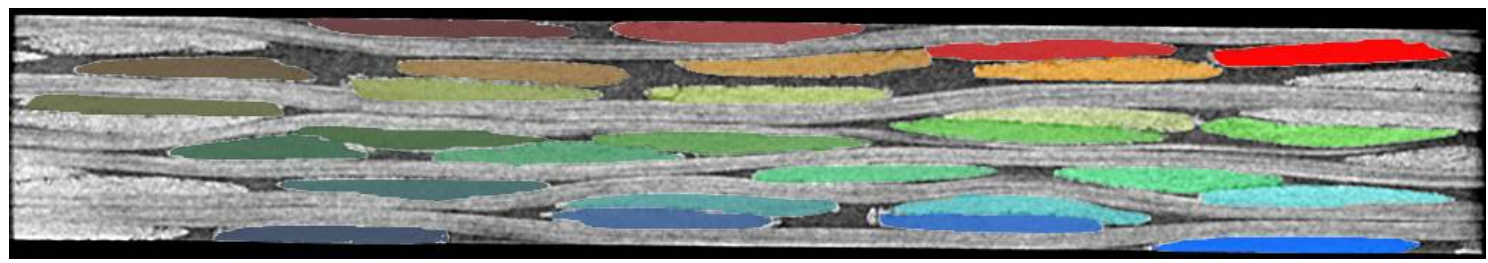

Figure 4: Post-processed image of a cross-section of the composite sample (one unit cell, 7 layers)

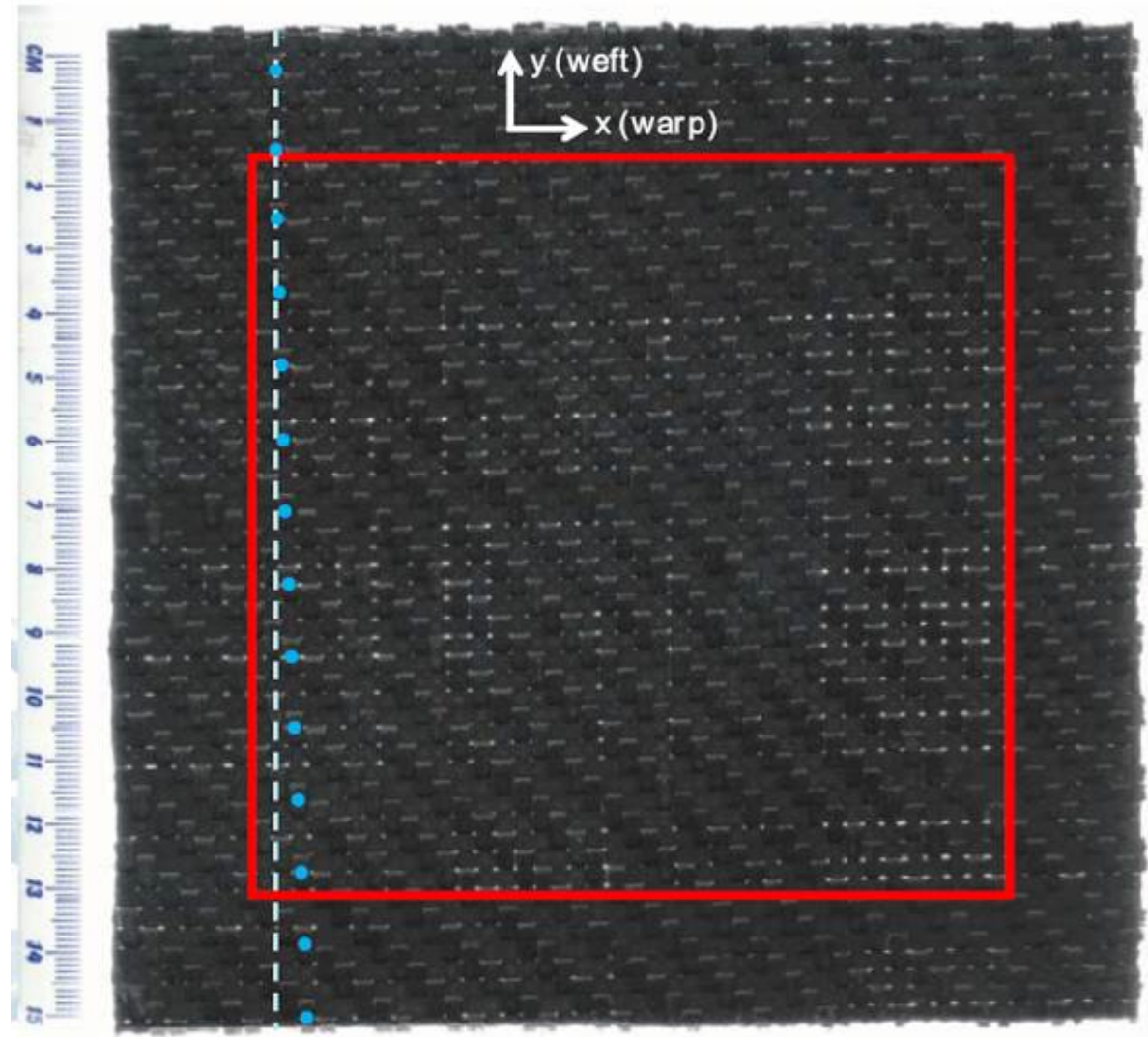

Figure 5: Image of an optical scan of the composite sample (one single layer)

\subsubsection{Geometry data acquisition}

Two sets of measurements have been conducted on two different types of samples of the composite:

- For the characterisation of short-range scatter $\mu \mathrm{CT}$ scans have been taken on samples with dimensions close to the unit cell size. These samples have 7 layers. Figure 4 shows a postprocessed image of one cross-section. Colours indicate the tows which are intersected. Olave et al. [23] discuss the details of this measurement campaign.

- For the characterisation of longe-range scatter optical scans have been taken on samples with dimensions equal to several unit cell size. These samples have only one layer. Figure 5 shows an image of an optical scan. 


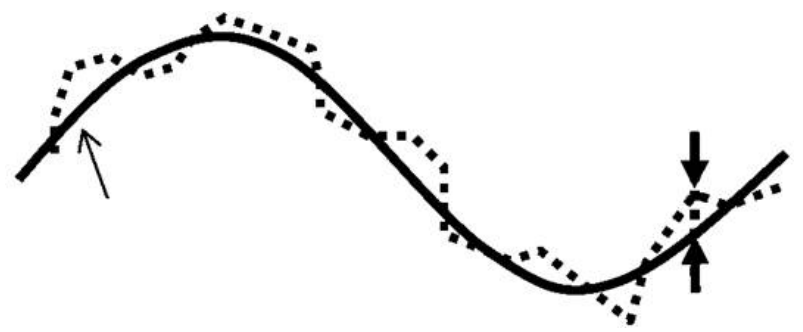

Figure 6: Representation of the out-of-plane position of the tow centroid over one unit cell: the solid line represents the average trend and the dotted line shows local variations with respect to the average trend

\subsubsection{Statistical processing of tow data}

Statistical analysis is conducted on the date of each individual tow. Figure 6 shows the result, for variations of the out-of-plane centroid position. The average trend corresponds to the first term in equation (1) and the deviation is represented by the second term in the equation. The average trend in the figure exhibits a wavy pattern, marking the crossing of one warp tow over or under a weft tow.

\subsubsection{Modelling of tow data}

In the modelling step, models are generated which extend over 10 unit cells, with the length of one unit cell being represented by 32 equi-distant points. Short-range periodic trends and long-range handling trends are combined. Figure 7 shows the trends for the warp and weft genus, for each of the five tow attributes which are measured. It was found that simulated trends correspond to experimental trends when cross-correlation is taken into account. Figure 8 compares the lateral deviation of the warp tow centroid. A very good match between simulations and experiments is found.

\subsubsection{Generation of virtual models}

In the final step, 3D models are generated in the WiseTex pre-processor. Figure 9 shows models of one unit cell and one larger sample. The reader should pay attention to the spacings between tows. The figure shows clearly that spacings in the generated virtual models are irregular.

\section{CONCLUSIONS}

This research is an effort to develop physically realistic virtual models of real carbon fibre reinforced composite samples. The approach consists of three successive phases: (1) the collection of spatial experimental data with complete statistical analysis, including short-range (meso-scale) geometrical scatter and long-range (macro-scale) geometrical variation, and statistical analysis in terms of average trend, standard deviation and correlation length, (2) stochastic multi-scale modelling of the reinforcement, including the definition of systematic and handling trends as they are extracted from the experimental data, and (3) the construction of virtual specimens in the WiseTex pre-processing software.

The methodology is demonstrated for a carbon-epoxy $2 / 2$ twill woven composite. The comparison between experimental and simulated deviation trends is found to be good. Randomly generated virtual specimens possess the target statistical information. 

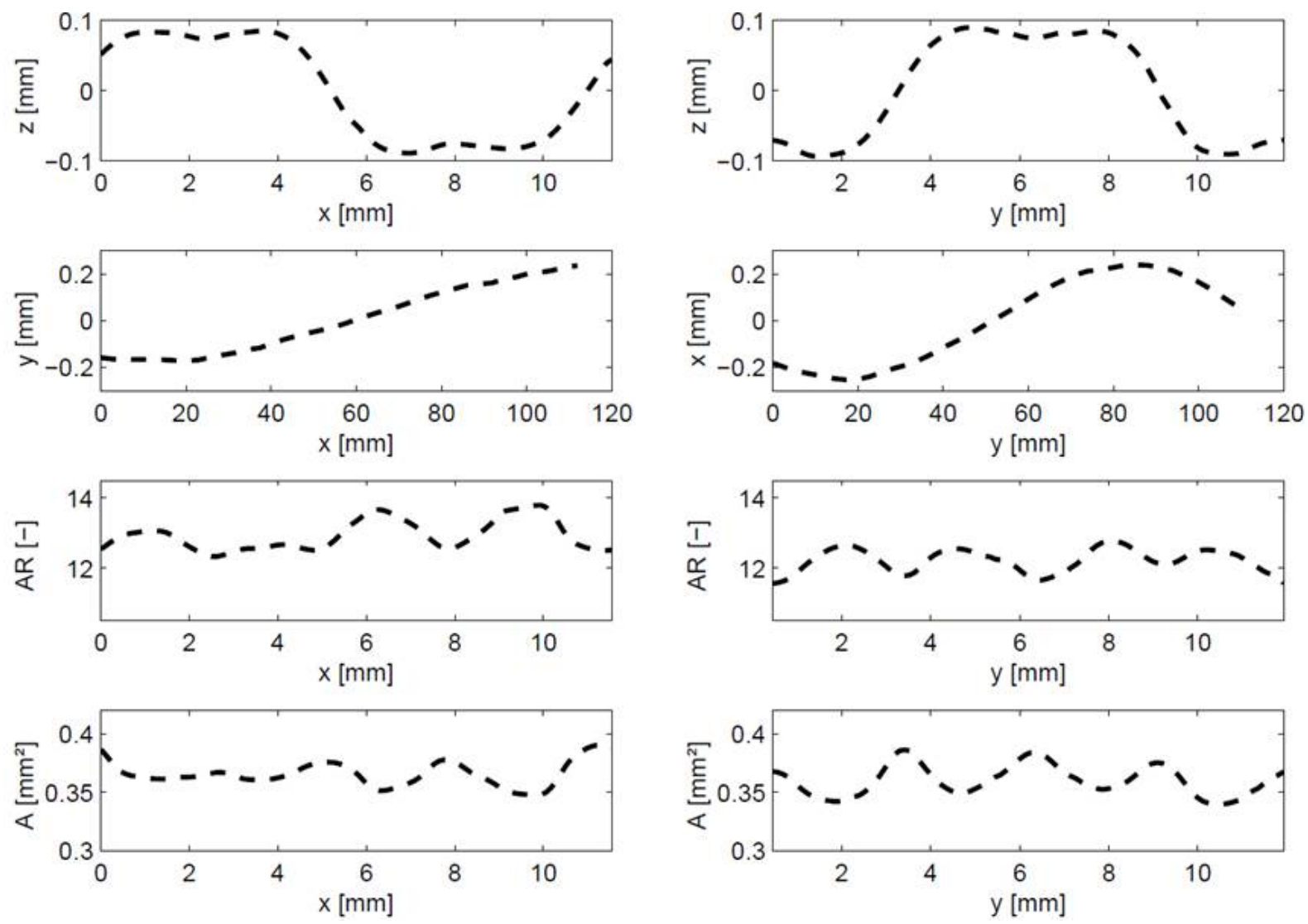

Figure 7: Trends on each of the tow attributes: the left hand graphs apply for the warp genus, and the right hand column applies to the weft genus; the top line graphs show the out-of-plane position of the tow centroid for one unit cell; the second line graphs represent the lateral in-plane deviation of the tow centroid for 10 unit cells; the third and fourth line graphs show the aspect ratio and the cross-section, respectively, each for one unit cell 

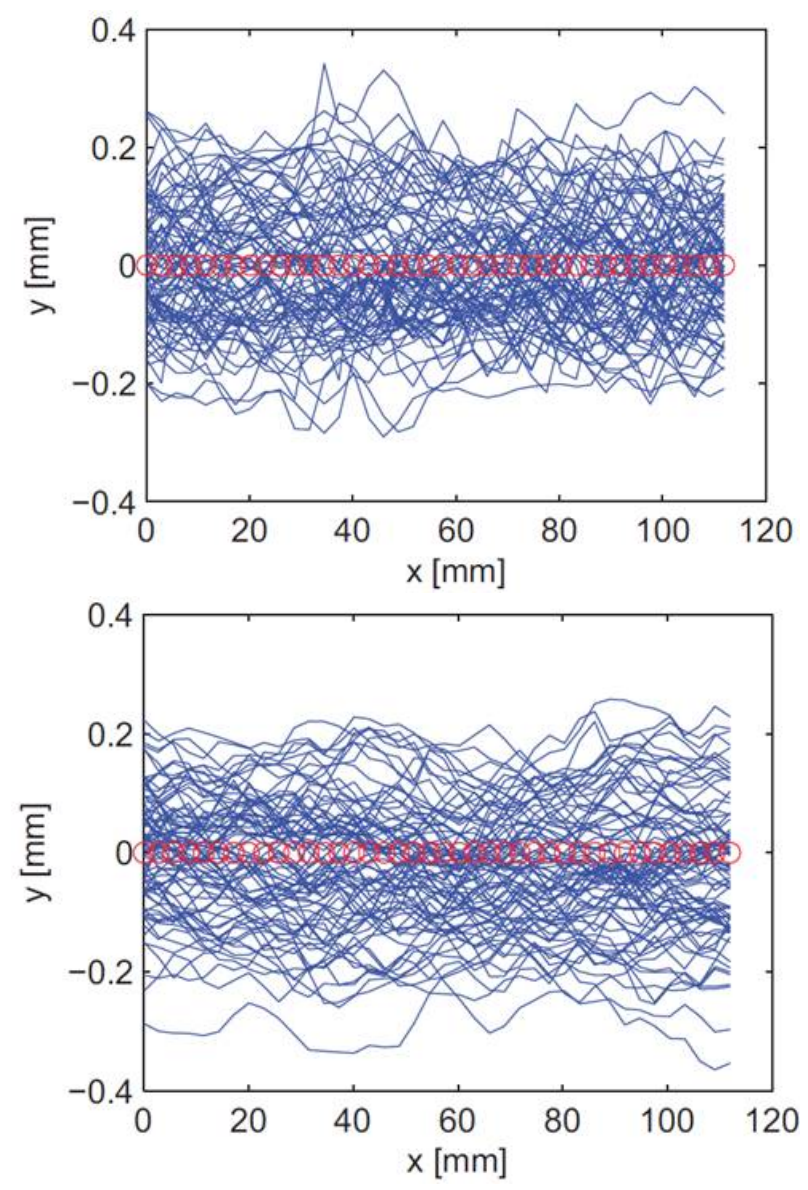

Figure 8: Comparison of lateral deviations trend for the warp tow: left $=$ experimental - right $=$ simulated

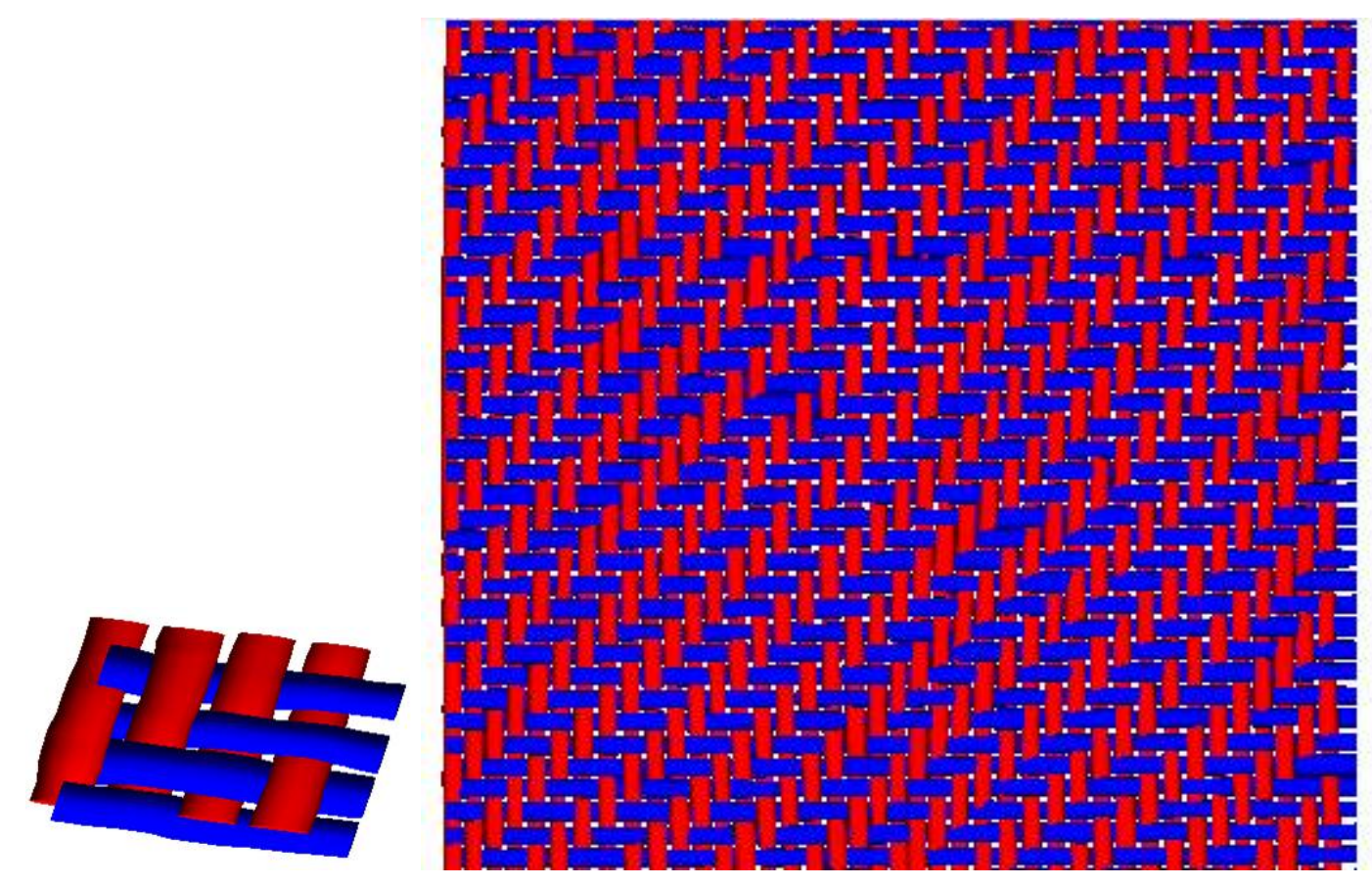

Figure 9: WiseTex generated models of one layer of the composite; left: one unit cell — right: larger sample 
In a next phase of research, these models will be used to identify the effect of intra-sample geometrical variability on homogenised properties of a composite panel.

\section{ACKNOWLEDGEMENT}

The authors gratefully acknowledge the agencies which have funded this research: FWOVlaanderen (Flemish Fund for Scientific Research) and IWT-Vlaanderen (the Flemish agency for the transfer of research results to industry).

\section{REFERENCES}

[1] D. Vandepitte, D. Moens, Quantification of Uncertain and Variable Model Parameters in Non-Deterministic Analysis, IUTAM 2009, IUTAM Symposium on the Vibration Analysis of Structures with Uncertainties, 27, 71-83, 2011.

[2] A. Freudenthal, Fatigue sensitivity and reliability of mechanical systems, especially aircraft structures, WADD Technical Report 61-53, 1961

[3] S. Langelaan, S. Deprez, I. Verpoest, P. Van Houtte, Determination of morphological textures of the fibres in composite materials made from textiles of carbon fibres, Materials Science Forum, 495-497, 1675-1680, 2005.

[4] J. Zeman, M. Sejnoha, Homogenization of balanced plain weave composites with imperfect microstructure: Part I - Theoretical formulation, International Journal of Solids and Structures, 41, 6549-6571, 2004

[5] M. Sejnoha, J. Zeman, Overall viscoelastic response of random fibrous composites with statistically quasi uniform distribution of reinforcements, CMAME, 191, 44, 5027-5044, 2002

[6] F. Desplentere, S.V. Lomov, D.L. Woerdeman, I. Verpoest, M. Wevers, A. Bogdanovich, Micro-CT characterization of variability in $3 D$ textile architecture, Composites Science \& Technology, 65, 1920-1930, 2005

[7] A. Endruweit, A.C. Long, F. Robitaille, C.D. Rudd, Dependence of permeability variations on the textile structure, Proceedings ECCM-11, June 2004

[8] B. Markicevic, D. Heider, S.D. Advani, S. Walsh, Stochastic modeling of preform heterogeneity to address dry spots formation in the VARTM Process, Composites part A, 36, $851-858,2005$

[9] E.H. Vanmarcke, MIT Press, Cambridge, Massachussetts, London, 1983

[10] C.C. Li, A. Der Kiureghian, Technical Report UCB/SEMM-92/04, University of California, Berkeley, 1992

[11] D.B. Chung, M.A. Gutierrez, R. de Borst, Object-oriented stochastic finite element analysis of fibre metal laminates, CMAME, 194, 12-16, 1427-1446, 2005

[12] D.C. Charmpis, G.I. Schuëller, M.F. Pellissetti, The need for linking micromechanics of materials with stochastic finite elements: a challenge for materials science, Computational Materials Science, 41, 1, 27-37, 2007 
[13] P.K. Mallick, Fiber-reinforced composites: Materials, Manufacturing and Design, 3rd edition, CRC Press - Taylor \& Francis Group, 2008

[14] M. Kawai, N. Honda, Off-axis fatigue behavior of a carbon/epoxy cross-ply laminate and predictions considering inelasticity and in situ strength of embedded plies, International Journal of Fatigue, 30 (10-11), 1743-1755, 2008

[15] H. Bale, M. Blacklock, M.R. Begley, D.B. Marshall, B.N. Cox, R.O. Ritchie, Characterizing three-dimensional textile ceramic composites using synchrotron $x$-ray microcomputed-tomography, Journal of the American Ceramic Society, 95(1),392-402, 2012

[16] A. Vanaerschot, B. Cox, S.V. Lomov, D. Vandepitte, Stochastic multi-scale modelling of textile composites based on internal geometry variability, Computers \& Structures, 122, 55-64, 2013.

[17] M. Blacklock, H. Bale, M. Begley, B. Cox, Generating virtual textile composite specimens using statistical data from micro-computed tomography: $1 D$ tow representations for the Binary Model, Journal of the Mechanics and Physics of Solids, 60(3), 451-470, 2012

[18] M. Vorechovský, Simulation of simply cross correlated random fields by series expansion methods, Structural Safety, 30(4), 337-363, 2008

[19] M. Vorechovský, D. Novák, Correlation control in small-sample Monte Carlo type simulations 1: A simulated annealing approach, Probabilistic Engineering Mechanics, 24(3), 452-462, 2009

[20] A. Vanaerschot, B. Cox, S.V. Lomov, D. Vandepitte, Stochastic framework for quantifying the geometrical variability of laminated textile composites using micro-computed tomography, Composites A, 4, 122-131, 2013.

[21] A. Vanaerschot, B. Cox, S.V. Lomov, D. Vandepitte, Simulation of the cross-correlated positions of in-plane tow centroids in textile composites based on experimental data, Composite Structures, 116, 75-83, 2014.

[22] http://www.mtm.kuleuven.be/Onderzoek/Composites/software/wisetex

[23] M. Olave, A. Vanaerschot, S.V. Lomov, D. Vandepitte, Internal geometry variability of two woven composites and related variability of the stiffness, Polymer Composites, 33 (8), 1335-1350, 2012 